\title{
¿Sexualidad ortodoxa o heterodoxa? La sexualidad en España en el siglo XXI ${ }^{1}$
}

\author{
Helena Pérez de la Merced \\ Universidad Autónoma de Madrid, Madrid, España \\ elena.perezd@uam.es
}

\begin{abstract}
Resumen: La sexualidad planea por doquier y, sin embargo, la sociología ha vuelto su mirada hacia esta área recientemente; algo aún más notable en el caso de nuestro país, donde los trabajos a este respecto han sido eminentemente teóricos e imbuidos de las creencias que dominaban el panorama político-social del momento. El presente trabajo pretende contribuir al estudio de las concepciones y comportamientos de la población española residente hacia la sexualidad a través de la búsqueda de factores explicativos de la misma. Este hecho representa la principal novedad de la presente investigación al intentar analizar cómo es nuestra sexualidad a través de la detección de dimensiones subyacentes, es decir, hacer medible el concepto de sexualidad dentro de los ejes normativo/no-normativo. Entre los resultados obtenidos, en una primera aproximación, se perciben diferencias en función de variables como el sexo, la edad y las creencias religiosas.
\end{abstract}

Palabras clave: sexualidad, conducta sexual, análisis factorial, ANOVA.

\begin{abstract}
Sexuality is everywhere but Sociology pays attention to it recently, especially in our country case where works in this area has been mainly theoretical based on the historical moment beliefs. This article aimed to contribute to the study of conceptions and behaviors of Spaniards towards sexuality through explanatory factors of it. This research aims to analyze how is our sexuality detecting underlying dimensions, in other words, make measurable the concept of sexuality on an axis of normative/ non-normative sexuality. Key findings show, in a first glance, differences regarding sex, age and religious beliefs are found.
\end{abstract}

Keywords: sexuality, sexual behavior, factor analysis, ANOVA.

${ }^{1}$ Trabajo galardonado con el Premio Jóvenes Sociólogos/as de la Asociación Madrileña de Sociología 2018. 


\section{INTRODUCCIÓN}

La sexualidad se encuentra por todas partes, empero, la sociología se ha ocupado de este campo hace escasas décadas —algo especialmente observable en nuestro paísEn este sentido, a la hora de realizar estudios que aborden el fenómeno de la sexualidad han primado estudios de corte teórico (De Ussel, 1983) con un marcado déficit de investigaciones de carácter empírico a través de análisis más sofisticados. Así pues, el presente trabajo nace con la pretensión de contribuir a llenar este vacío de conocimiento de la mano de una de las encuestas realizadas por el Centro de Investigaciones Sociológicas, la Encuesta Nacional sobre Salud Sexual del año 2008.

El objetivo de esta investigación estriba en identificar las principales dimensiones subyacentes de las concepciones sobre sexualidad que, debido a su naturaleza, no son directamente observables. Una vez realizada esta tarea, el siguiente objetivo es analizar la existencia de posibles diferencias significativas en la concepción sobre sexualidad en la población española residente en función del sexo, la edad y las creencias religiosas.

En el primer apartado, "Estado de la cuestión", se realiza una aproximación teórica al concepto de su sexualidad y a los diferentes modelos de la misma. En el segundo apartado, denominado "Metodología", se procede a describir la base de datos empleada y se presentan las variables incluidas en el análisis así como diferentes consideraciones sobre el tamaño de la muestra y las novedades de esta base de datos. En la tercera sección, "Análisis", se explica el proceso de análisis de los datos así como los resultados obtenidos. Por último, en las "Conclusiones" se evalúan dichos resultados, la aportación de la presente investigación y las futuras líneas de acción.

\section{ESTADO DE LA CUESTIÓN}

\section{Breve historia de la sexualidad}

El sexo es una actividad social y, como tal, posee normas. Cualquier estudio sobre sexualidad que se precie — como advierte Guasch (1993) — debe transitar la senda de la revisión de la manera en que se ha organizado el control de dicha actividad en Occidente. Así pues, el relato sobre la sexualidad acaecido a lo largo de la historia ha sido narrado a través de tres grandes perspectivas: la religión, la medicina y la sexología; y a su vez, todas ellas inscritas en diferentes periodos históricos: el Antiguo Régimen (religión), la Edad Moderna (medicina) y el Mundo Contemporáneo (sexología) (Guasch, 1993; Moreno, 1990). Desde un marcado punto de vista foucaultiano, podemos afirmar 
que la influencia de la religión, de la medicina o de la sexología en cada uno de estas tres etapas ha dado lugar a un determinado discurso hegemónico sobre la sexualidad.

Empezando por la tradición judeocristiana, esta concibe aún hoy el sexo dentro de la institución del matrimonio heterosexual, prohíbe el adulterio así como las relaciones sexuales antes del matrimonio, condena los actos homosexuales y tiene como principal fin la procreación (Masters, Johnson y Kolodny, 1995). Avanzando en el tiempo, en el siglo xix, el modelo de normalidad sexual es definido por la medicina. Esta traza un modelo de sexualidad eminentemente heterosexual, reproductiva y moral (Guasch, 1993). Por último, la sexología comienza su andadura a finales del siglo xix de la mano de autores como Freud o Kraft-Ebing con un origen estrictamente médico y centrado en las "desviaciones" (Moreno, 1990).

Si realizamos una comparación entre las tres perspectivas mencionadas, observamos que tanto el cristianismo como la medicina poseen una concepción coitocéntrica ${ }^{2}$ de la sexualidad. De igual manera, la sexología coloca el coito en el centro de toda relación sexual ${ }^{3}$, y aquello que lo dificulta es concebido como un problema en las relaciones sexuales. Así, por ejemplo, los pioneros del estudio de la sexualidad Masters y Johnson elaboran un exhaustivo listado de "trastornos sexuales del hombre y de la mujer" que en definitiva no son más que problemas u obstáculos para la realización del coito: impotencia, eyaculación precoz, vaginismo, anorgasmia, coito doloroso, etc. (Masters, Johnson y Kolodny, 1995). Ahondando en las semejanzas, desde un análisis de género, el cristianismo niega la sexualidad femenina, mientras que la medicina, por su parte, la juzga e interpreta en términos masculinos. Por su parte, la sexología reconoce el deseo sexual de la mujer, aunque cabe mencionar que lo realiza desde una perspectiva masculina. Por tanto, todas ellas se mueven dentro del espectro androcéntrico que tiene al varón como medida de todas las cosas. Por otro lado, tanto la medicina como el cristianismo condenan las disidencias sexuales, y aunque la sexología suprime el nombre de perversión del DSM y pasa a llamarlas parafilias (sadismo, voyerismo, pedofilia...), lo cierto es que sigue considerando estas conductas sexuales como prácticas alejadas de la norma. Por último, el cristianismo y la medicina defienden el sexo dentro del matrimonio, y pese a que la sexología, por su parte, no lo limita

${ }^{2}$ Coito: penetración del pene estando erecto en la vagina y/o el ano (CIS, 2009: 26). En este caso, nos referimos al coito vaginal. Por coitocentrismo nos referimos a la creencia que tiende a colocar el coito en el centro de la relación sexual, restando importancia al resto de posibles prácticas sexuales.

${ }^{3}$ En este sentido, es frecuente el uso de términos como "relaciones completas" cuando ha existido coito; "preliminares", prácticas anteriores que preparan para la actividad sexual principal, el coito; o hablar de "pérdida de la virginidad", cuando una persona ha practicado el coito vaginal por primera vez.. 
al ámbito conyugal, defiende la pareja como el escenario ideal de la actividad sexual (Guasch, 1993: 115). Prueba de ello es que, desde los pioneros en terapia sexual, Masters y Johnson, esta se desarrolla en pareja y no tratando a individuos aislados (Masters, Johnson y Kolodny, 1995).

En suma, primero el cristianismo se ocupa de establecer los pecados, más tarde la medicina define los atentados contra la moral y, por último, la sexología — cuya misión es similar a las dos anteriores - se encarga de dar normas al sexo y estipular qué es lo correcto e incorrecto en relación con las prácticas sexuales. Una vez andado el camino de la regulación de la sexualidad en Occidente a lo largo de la historia, podemos concluir que esta posee características comunes en todas las etapas: la defensa del matrimonio o la pareja estable, la hegemonía del sexo coitocéntrico y genital y la definición de la sexualidad en términos masculinos (Guasch, 1993).

\section{El estudio de la sexualidad}

Centrándonos ahora en el estudio de la sexualidad, este se ha movido en torno a dos ejes: en un extremo, un encuadre biologicista profundamente guidado por disciplinas como la medicina y la psiquiatría, y, del otro lado, un segundo enfoque construccionista que ha mirado hacia aspectos histórico-culturales para poner de relieve la dimensión social de la sexualidad.

El gran giro construccionista se da durante las décadas de 1960 y 1970, cuando se plantea que las sexualidades pueden ser estudiadas sociológicamente. Hasta estas décadas resulta hegemónica la definición de la sexualidad como elemento presocial y biológico; asumiendo su carácter natural y, por consiguiente, negando la influencia de mecanismos sociales, políticos y económicos que moldean la sexualidad. Esta visión esencialista entra en crisis, y empieza a forjarse así la teoría social de la sexualidad (Osborne y Guasch, 2003). Por consiguiente, si aceptamos que la sexualidad no es inmutable, debemos dar por sentado que su estudio debe ser necesariamente abordado desde un punto de vista sociológico. Desde esta perspectiva, la sociología de la sexualidad incluye el sexo en tanto que actividad social, se ocupa de definir qué es sexo y qué no lo es, describe qué espacios y qué tiempos tiene adjudicados, qué actores lo ejecutan y cuáles no, de qué modo lo hacen, así como las razones y consecuencias sociales de todo ello (Guasch, 1993; Osborne y Guasch, 2003).

Entre estas primeras aportaciones que desafían el enfoque esencialista encontramos a académicas feministas como Firestone, Millet, Radicalesbians o Rubin que poseen como base argumental la separación de los conceptos de sexo y género, generando así 
la progresiva deconstrucción de estereotipos sobre la sexualidad del hombre y de la mujer (Bernstein, 2013). En el marco de esta perspectiva construccionista, la sexualidad comienza a ser estudiada desde una dimensión de poder - influida por la impronta foucaultiana- que necesita ser teorizada en términos políticos (Bernstein, 2013). Por ejemplo, en contraposición con el discurso que durante los 60 venía de la mano de autores como Freud y Kinsey, que trataban la homosexualidad como desviación, esta primera generación de construccionistas (Weeks, Plummer) coloca las "desviaciones" dentro de los marcos de poder (Stein y Plummer, 1994). En este contexto se enmarca Rubin (1989) —artífice del sistema sexo/género—, elaborando una suerte de jerarquía sexual que constituye una herramienta teórica que desvela el hecho de que las sociedades occidentales modernas evalúan los actos sexuales según un sistema jerárquico de valor sexual.

Según la autora, la sexualidad buena, normal y natural es heterosexual, reproductiva y no comercial; se da dentro del matrimonio, es monógama y en parejas; se realiza entre personas de la misma generación y se practica en los hogares. Fuera de este marco normativo quedan la pornografía, los objetos fetichistas, los juguetes sexuales de todo tipo y cualesquiera otros papeles que no fueran el de macho y hembra. El sexo malo, anormal o antinatural es homosexual, promiscuo, sin un fin procreador, comercial o situado fuera del matrimonio (prácticas como la masturbación, las orgias...), cruza las fronteras generacionales, se practica en público y usa la pornografía, los objetos fetichistas, los juguetes sexuales o roles distintos a los tradicionales. Sin embargo, cabe mencionar que la masturbación y ciertas formas de homosexualidad (monógama) se mueven hacia la respetabilidad (Rubin, 1989).

Frente a esta visión, autores como Giddens han llamado la atención sobre el cambio acaecido en el terreno de la sexualidad durante las últimas décadas en nuestras sociedades occidentales. Giddens (1995) acuña el término "sexualidad plástica" para describir cómo la sexualidad ha sido liberada de la carga de la reproducción. Asimismo frente al modelo de amor romántico el autor habla del "amor confluente", que es "un amor que choca con las expresiones de 'para siempre', 'solo y único', que se utilizan por el complejo amor romántico", con un papel de sexos más igualitario y con una menor identificación proyectiva con el/la otro/a (Giddens, 1995: 63). Otra de las observaciones del autor viene a llamar la atención sobre el excesivo énfasis en el poder y en el discurso de estudios como el de Foucault, en los que la historia hecha por los individuos como sujetos se desvanece.

A raíz de esto último cabe preguntarse: ¿todos los comportamientos y actitudes sexuales obedecen a mandatos sociales? ¿Podemos predecir los mismos exclusivamente a partir de escenarios culturales? 
En este punto rescataremos otra de las grandes contribuciones a la sociología de la sexualidad: la de John Gagnon y William Simon, pioneros en la aplicación de los conceptos sociológicos a la variedad erótica y punto de referencia de la perspectiva construccionista en el estudio de la sexualidad. Estos autores acuñaron el concepto de guion sexual (Gagnon y Simon, 1973) afirmando que la sexualidad no flota libre, sino guiada por fuerzas materiales y culturales y por la interacción entre lo social y lo personal. En este sentido, las actividades sexuales han de ser entendidas como el resultado de un complejo proceso psicosocial de desarrollo compuestos por guiones personales, guiones interpersonales y guiones histórico-culturales o sociales.

Los guiones sociales (Gagnon y Simon, 1973, 2003, 2011; Wiederman, 2015) serían aquellos escenarios culturales que colocan en el campo de la sexualidad lo que es considerado como deseable y no deseable, es decir, dónde están los límites entre la conducta sexual apropiada e inapropiada. Los guiones sociales por sí mismos no nos explican el comportamiento interpersonal en determinadas situaciones, y es aquí donde los guiones interpersonales entran, esto es, la organización de convenciones mutuamente compartidas que permite a uno o más actores participar en un acto complejo que conlleva mutua dependencia. Los guiones personales o intrapsíquicos representan las particularidades de cada sexualidad única de cada individuo. Cabe mencionar que estos tres niveles se encuentran dinámicamente relacionados. Por ejemplo, los autores observan que los guiones sexuales están conectados con los guiones de género y con ciertas asunciones producto de la cultura y la socialización: por ejemplo, de la socialización de género que afecta a guiones personales y que luego se reproduce en los guiones interpersonales (Simon y Gagnon, 2011).

Gagnon y Simon (1973), desde un punto de vista dramatúrgico, presentan al sujeto como autor en el nivel intrapsíquico, como actor en el escenario intrapersonal y como espectador, crítico y corrector en el escenario cultural. Así pues, los individuos reproducen guiones personales adaptándose a los escenarios culturales internalizados (Laumann et al., 2000).

Dentro de los guiones de género, la sexualidad es una construcción social basada en relaciones de poder donde se representa a los hombres como físicamente fuertes y activos y a las mujeres como personajes más pasivos. Así, la heterosexualidad construye una jerarquía de género basada en definiciones de lo que significa ser hombre y ser mujer junto con una serie de suposiciones sobre cómo se deben tener relaciones sexuales. En su función institucional social, la heterosexualidad fomenta pautas culturales como el noviazgo y el romance heterosexual (Macionis y Plummer, 2012: 352-353). Según los autores, hombres y mujeres aprenden diferentes guiones 
debido a la socialización de género, el típico guion sexual del hombre incluye una búsqueda activa de sus compañeros y compañeras sexuales, poca habilidad para controlar el impulso sexual y el sexo en solitario en la constante búsqueda de placer. La mujer, por su parte, espera a ser escogida en lugar de practicar la búsqueda activa y las relaciones sexuales se encaminan a la búsqueda de afecto y amor (Frith y Kitzinger, 2001; Alkins 1996).

Dentro de este esquema, el concepto del amor romántico es un instrumento de manipulación especialmente para la mujer, ya que el amor es lo que autoriza la relación sexual de la mujer (Millet, 1995). Así pues, esperaremos que las mujeres posean una visión más normativa del sexo en relación con el amor que los hombres. Así mismo, los hombres y niños son vistos como naturalmente más activos sexualmente (conducidos por sus impulsos) que las niñas y las mujeres (Macionis y Plummer, 2012: 352-353). Sin embargo, otras posturas apuntan a que la modernidad ha ido debilitando este imaginario y la mujer ha empezado a reconocerse también en el ejercicio de su sexualidad (Giddens, 1995).

La revisión del fenómeno de la sexualidad nos acerca, por tanto, a la idea de que existen dos concepciones sobre la sexualidad. Es decir, dos modelos: uno de ellos de corte más tradicional y otro modelo alternativo a este. Todas sus características vendrían definidas en la Figura 1.

\section{FIGURA 1}

Modelos de sexualidad: ortodoxa y heterodoxa

\section{Sexualidad ortodoxa/normativa}

Defensa del matrimonio y/o de la pareja

estable/monogamia

Coitocentrismo y genitalidad

Androcentrismo (definición de la sexualidad en términos masculinos)

Heterosexualidad

Noviazgo y romance heterosexual, amor romántico

Género de esquema binario

Sin uso de elementos ajenos al propio cuerpo

Parafilias

Tradición judeocristiana

\section{Sexualidad heterodoxa/no-normativa}

Relaciones no convencionales (poliamor, no-monogamia...)

Sexualidad no-coitocéntrica y holística

Sexualidad femenina

Diversidad en la orientación sexual

Poliamor, no monogamia, anarquía

relacional, amor confluente...

Roles distintos a los tradicionales

Pornografía, objetos fetichistas, juguetes...

Diversidad de deseos

Desheredada de la tradición judeocristiana

Fuente: Elaboración propia a partir de la revisión teórica. 


\section{Estudios sobre sexualidad en España}

Cuando la sociología trata el tema de la sexualidad - desde Freud hasta Masters y Johnson-, lo hace pensando en una sociedad victoriana diferente de la española y su especial capacidad represiva en este aspecto (De Miguel, 1975: 55). Desde la perspectiva sociológica, los únicos estudios con contenido sexual que se llevaron a cabo en nuestro país fueron aquellos relacionados con análisis demográficos y con las encuestas sobre control de la natalidad. Asimismo se incluían algunas preguntas sobre sexualidad en la Revista Española de Opinión Pública, en la revista del Instituto de la Juventud y en la prensa. Si tuviéramos que elaborar rasgos comunes de todas ellas, diríamos que medían actitudes y no tanto comportamientos, abordaban la sexualidad de manera individual y no en pareja, prestaban más atención a sexualidad prematrimonial que a la matrimonial, concentraban estudios sobre jóvenes y no sobre adultos, se centraban en la heterosexualidad y aparecían vinculados al control de la natalidad (De Ussel, 1983). En este sentido, De Ussel llamaba la atención sobre "la recapitulación de los conocimientos adquiridos sobre la sexualidad en nuestro país como paso previo al impulso definitivo de la sociología de la sexualidad, refugiada todavía en el ensayismo" (De Ussel, 1983: 131). Según Guasch (1993), diez años después, el estado de las investigaciones sociológicas sobre sexo reproduce las características que ya señaló De Ussel en 1983.

Este vacío se ha ido llenando aunque lentamente, puesto que la sociología de la sexualidad sigue sin ser uno de los campos clave dentro de la sociología en España. Uno de los estudios recientes sobre sexualidad en nuestro país es el realizado por Ayuso y García (2014), el cual utiliza dos bases de datos: la de los estudios Actitudes y Prácticas Sexuales (CIS, 2738) y la Encuesta Nacional sobre Salud Sexual usada en la presente investigación (CIS, 2780). Este estudio posee un carácter eminentemente descriptivo a través de análisis de frecuencias, medias y tablas de contingencia, ampliado en algunos casos con análisis de segmentación y escogiendo como variables independientes el sexo, la edad, el nivel de estudios, el tamaño del hábitat de residencia, el autoposicionamiento o la práctica religiosa (Ayuso y García, 2014).

Entre los hallazgos, Ayuso y García (2014) concluyen que se ha producido en las últimas décadas una "transición sexual", es decir, un aumento de la tolerancia a la hora de percibir la vida íntima pese a que el peso de la tradición cultural católica no ha permitido realizar una "revolución sexual" tan rotunda como en otros países europeos. Esta "transición sexual" obedece a cinco factores clave según los autores: 1) el proceso de secularización; 2) los cambios familiares a la hora de construir la intimidad (amor más individual y negociado); 3) erotización de la vida cotidiana; 4) mayor libertad para optar por una orientación sexual e identidad de género diferentes a las normativas; y 5) la 
concepción de la sexualidad como forma de comunicación (Ayuso, 2015: 335). En 2011, y bajo el título "Jóvenes y diversidad sexual", se publicó un estudio cualitativo a través de grupos de discusión que concluía que entre los jóvenes existía una mayor tendencia hacia la aceptación de una mayor plasticidad de las identidades sexuales y de un mayor reconocimiento de la existencia de la diversidad afectivo-sexual (INJUVE, 2011). No obstante, otros autores nos dirán que se sexualiza el mundo, pero se comercializa y objetualiza el cuerpo femenino - recordando a MacKinnon (1979) la "cotidiana erotización de la subordinación de las mujeres"-, que la homosexualidad sigue sin ser reconocida como una opción legítima y que la heterosexualidad normativa y monógama continúa en el eslabón más alto de la jerarquía sexual (Osborne y Guasch, 2003). Por tanto, el presente estudio pretende dilucidar si perviven concepciones de la sexualidad más tradicionales con otras que se adhieren a esta "transición sexual".

En relación con el estudio de Ayuso y García (2014), uno de los hallazgos del análisis descriptivo es que existen diferencias entre las actitudes y los comportamientos sexuales entre las personas más jóvenes y las de mayor edad puesto que han sido socializadas en un contexto diferente porque nuestro país ha pasado de una cultura sexual en la que predominaba el miedo y se basaba en la moral católica a una llamada "erotización de la vida cotidiana". Por tanto, siguiendo esta línea, nuestra hipótesis es que las personas de mayor edad poseerán una concepción de la sexualidad más normativa que las jóvenes.

El mismo análisis de Ayuso y García percibía que existen diferencias entre hombres y mujeres a la hora de percibir su sexualidad como consecuencia de los procesos de socialización de género. Algo que camina en la línea de la teoría de los guiones sexuales: los guiones sexuales están conectados con los guiones de género y ciertas asunciones son productos de la cultura y la socialización; dentro de esto, la socialización de género afecta a los guiones personales que a su vez se reproducen en los guiones interpersonales (Gagnon y Simon, 2011). Nuestra hipótesis, por tanto, es que las mujeres poseerán una concepción más ortodoxa de la sexualidad de lo que lo harán los hombres.

Asimismo Ayuso y García detectaron en sus análisis descriptivos que la religión influye en la vivencia de la sexualidad, reforzándose una concepción más tradicional de aquella. Es decir, las personas con creencias religiosas poseerán una concepción más ortodoxa de la sexualidad que las que no las tienen.

Por tanto, los objetivos de la investigación son: 1) identificar las principales dimensiones subyacentes de las concepciones sobre sexualidad que, debido a su naturaleza, no son directamente observables y, por otro lado; 2) analizar si existen diferencias significativas en la concepción de la sexualidad en la población española residente en función del sexo, la edad y las creencias religiosas. 


\section{METODOLOGÍA}

\section{Datos utilizados}

Los datos utilizados para la realización de la presente investigación proceden de la I Encuesta Nacional de Salud Sexual (ENSS) realizada por el Centro de Investigaciones Sociológicas (estudio CIS n. ${ }^{\circ}$ 2780) en colaboración con el Observatorio de Salud de la Mujer (OSM), del Sistema Nacional de Salud, del Ministerio de Sanidad y Política Social. La ENSS se enmarca en el I Plan de Calidad del Sistema Nacional de Salud para "promover el conocimiento sobre las desigualdades de género en la salud y el fortalecimiento del enfoque de género en las políticas de salud y el análisis de la salud sexual desde un enfoque de género" (CIS, 2009: 4). El objetivo de la ENSS estribaba en "obtener un primer diagnóstico de la situación a nivel estatal, de cara a la elaboración de una estrategia de salud sexual" (CIS, 2009: 26).

La encuesta se dirigió a población residente de ambos sexos de 16 años y más. El tamaño de la muestra se estableció en 10.000 entrevistas con un diseño proporcional para el cruce de las variables sexo y tramos de edad. La muestra final la componen 9.850 individuos por un procedimiento de muestreo polietápico, estratificado por conglomerados, con selección de las unidades primarias de muestreo (municipios) y de las unidades secundarias (secciones) de forma aleatoria proporcional, y de las últimas unidades (individuos) por rutas aleatorias y cuotas de sexo y edad.

En 2008 se realizó un estudio piloto, y entre los meses de noviembre y enero se llevó a cabo el trabajo de campo. Teniendo en cuenta el carácter íntimo y sensible de muchas de las preguntas, la encuesta se realizó mediante un modelo mixto de administración (autocumplimentado por la persona entrevistada y cumplimentado por el/la entrevistador $\left./ \mathrm{a}^{4}\right)$.

La ENSS resulta novedosa con respecto a encuestas anteriores que tratan el tema de la sexualidad y/o la salud o incorporan alguno de sus aspectos en lo relativo a su enfoque. En primer lugar, las encuestas previas nacionales ${ }^{5}$ no incluyen a población mayor

${ }^{4}$ Preguntas relativas a las primeras relaciones sexuales y a las relaciones sexuales en los doce últimos meses para posibilitar el análisis de las respuestas teniendo en cuenta el modo de administración.

${ }^{5}$ Encuesta Nacional de Salud (ENS) de 2006 y Encuesta de Salud y Hábitos Sexuales INE (ESHS) de 2003 del Instituto Nacional de Estadística (INE). Encuesta sobre Sexualidad en Jóvenes de la Comunidad Valenciana del año 2000. Encuesta irlandesa sobre Actitudes y Conocimientos Sexuales, la británica Encuesta Nacional de Actitudes Sexuales y Estilos de Vida (NATSAL) y el estudio francés sobre La Sexualidad en Francia. Prácticas, Género y Salud de 2006. 
de 65 años o más años. Y en segundo lugar, ofrecen una visión medicalizada de la sexualidad, por ejemplo, centrándose excesivamente en las enfermedades de transmisión sexual (ETS). Por último, cabe mencionar que todas ellas poseen una visión patriarcal de la sexualidad en su conjunto.

Cabe mencionar a este respecto que la ENSS posee un diseño muestral que posibilita el análisis para el conjunto de la muestra así como para submuestras, lo que permite realizar análisis por separado para cada sexo, grupos de edad o el cruce de sexo y edad. Asimismo todas las fases de este estudio se diseñaron desde una perspectiva de género teniendo en cuenta un concepto más holístico de lo que significa relación sexual ${ }^{6}$ y considerando las relaciones sexuales en sentido amplio: desde un enfoque no androcéntrico y no coitocentrista.

Por tanto, en lo que a la conceptualización de sexualidad normativa/no-normativa se refiere, la ENSS incluye indicadores que cubren las dimensiones que la literatura nos ofrece a este respecto. Los indicadores de sexualidad normativa/no-normativa están clasificados en cuatro grandes dimensiones en el cuestionario original, en el que se distinguen diferentes bloques que responden a objetivos específicos: en el Anexo 1 se detallan cuáles han sido elegidos para hacer operativo el concepto de sexualidad normativa/no-normativa.

\section{Método}

Uno de los objetivos de la presente investigación es identificar las principales dimensiones subyacentes de las concepciones sobre sexualidad que, debido a su naturaleza, no son directamente observables. Una vez obtenidas estas dimensiones, se pretende explicar, en una primera aproximación, cómo inciden diferentes variables sociodemográficas (sexo, edad y creencias religiosas) en la concepción y vivencia de la sexualidad dentro del eje normativa/no-normativa.

Teniendo lo anterior presente, la técnica pertinente para cubrir el mencionado objetivo es el análisis factorial al permitir extraer la información contenida en un conjunto de variables para cuantificar las dimensiones subyacentes del concepto de sexualidad ortodoxa/heterodoxa que no pueden ser percibidas de manera directa. Por tanto, se reducirá la dimensión del análisis transformando un conjunto amplio de variables en un número más reducido de factores o dimensiones latentes de la sexualidad. Yendo más allá en el análisis, el valor de los factores obtenidos permitirá colocar a las personas a lo largo de este

${ }^{6}$ En este estudio se entiende por relación sexual al conjunto de prácticas y comportamientos que realizan dos o más personas, de igual o diferente sexo, en las que aparecen componentes de excitación y deseo sexual, con la finalidad de obtener placer sexual, que no tiene por qué concluir en coito. 
eje de sexualidad ortodoxa/heterodoxa. A partir de esta información, el segundo objetivo, por tanto, es analizar si existen diferencias significativas en la concepción de la sexualidad en la población española residente en función del sexo, la edad y las creencias religiosas. Para ello, se procederá a realizar un análisis de varianza (ANOVA) con el propósito de estudiar la incidencia de las diferentes variables sociodemográficas mencionadas anteriormente en las variables obtenidas (factores) como resultado del análisis factorial.

La elección de las variables escogidas para el análisis factorial ha estado basada en criterios sustantivos, utilizando aquellas variables que el cuestionario nos ofrece para realizar la conceptualización de sexualidad ortodoxa/heterodoxa.

Con respecto a las variables sociodemográficas para el análisis ANOVA, se han escogido para esta primera aproximación las siguientes: sexo, edad y creencias religiosas, teniendo en cuenta la literatura y los estudios previos a este respecto.

\section{ANÁLISIS}

\section{Descripción de los datos}

Para el análisis factorial se han elegido 23 variables que se corresponden con indicadores del concepto de sexualidad normativa/no-normativa que obedece a criterios sustantivos. Se trata de 23 variables de escala ordinal con categorías de respuesta estilo Likert (de 1 a 4 o de 1 a 5). Con respecto al tratamiento de las variables, las categorías de respuesta "NS" y "NC" se han tratado como casos perdidos. Por otro lado, con el objetivo de homogeneizar las variables en la escala sexualidad ortodoxa-heterodoxa, se ha realizado una transformación en la escala de alguna de las variables de manera que el valor numérico más bajo implica una sexualidad más ortodoxa y normativa, mientras que el valor más elevado indica una sexualidad más heterodoxa o no-normativa. Con esto último se facilita su tratamiento y análisis posterior una vez obtenidas las dimensiones tras la realización del análisis. El tamaño de la muestra analizada ha sido finalmente de 5.317 individuos, es decir, aquellas personas que respondieron a todas las preguntas incluidas en el análisis.

\section{Identificación de los factores subyacentes: análisis factorial}

Centrándonos ahora en el análisis factorial, se ha optado por el análisis de componentes principales (CP). Nuestro modelo explica proporciones de varianza suficientes ya que todas las comunalidades superan el valor del $80 \%$, confirmándose así que todos los indicadores están correctamente representados en los factores identificados. Además, los resultados del test Kaiser, Meyer y Olkin (KMO) permiten rechazar la hipótesis 
nula de ausencia de correlación entre los indicadores, lo que garantiza la robustez en los resultados del análisis factorial. Finalmente se seleccionaron un total de 6 factores, los cuales conjuntamente explican casi el $60 \%$ de la varianza total de la muestra.

Dado que todas las correlaciones o cargas factoriales tienen valores positivos, un valor alto en cada uno de los factores representará una concepción de la sexualidad más ortodoxa o normativa, mientras que los valores bajos se asociarán con una sexualidad heterodoxa o no-normativa. Los valores obtenidos en cada uno de los factores se han reescalado (en una escala de 0 a 10) con el objetivo de homogeneizar las mismas y facilitar su posterior interpretación y análisis.

TABLA 1

Matriz de componentes rotados (factor)

\begin{tabular}{lcccccc}
\hline & $\mathbf{1}$ & $\mathbf{2}$ & $\mathbf{3}$ & $\mathbf{4}$ & $\mathbf{5}$ & $\mathbf{6}$ \\
\hline \begin{tabular}{l} 
FACTOR 1. Prácticas no coitocéntricas \\
\hline P1902 Desnudar a su pareja.
\end{tabular} & 0,660 & 0,044 & 0,065 & 0,293 & $-0,024$ & 0,196 \\
$\begin{array}{l}\text { P1903 Que su pareja le desnude. } \\
\text { P1904 Acariciar a su pareja los }\end{array}$ & 0,663 & 0,065 & $-0,04$ & 0,28 & $-0,002$ & 0,204 \\
$\begin{array}{l}\text { genitales con manos, boca, lengua... } \\
\text { P1905 Que su pareja le acaricie los } \\
\text { genitales con manos, boca, lengua... }\end{array}$ & 0,780 & 0,069 & 0,166 & 0,163 & $-0,007$ & 0,071 \\
$\begin{array}{l}\text { P1906 Acariciar el cuerpo de su } \\
\text { pareja con manos, boca, lengua... }\end{array}$ & 0,862 & 0,103 & 0,139 & 0,172 & $-0,015$ & 0,069 \\
$\begin{array}{l}\text { P1907 Que su pareja le acaricie el } \\
\text { cuerpo con manos, boca, lengua... }\end{array}$ & 0,847 & 0,091 & 0,085 & $-0,005$ & 0,003 & $-0,02$ \\
\begin{tabular}{l} 
P1908 Besarse apasionadamente. \\
\hline
\end{tabular} & 0,660 & 0,082 & $-0,075$ & $-0,128$ & 0,008 & $-0,029$ \\
\hline
\end{tabular}

FACTOR 2. Actitudes hacia la orientación sexual diversa

\begin{tabular}{l|l|l|lllll}
\hline $\begin{array}{l}\text { P602 La relación sexual entre dos } \\
\text { mujeres es una opción personal tan } \\
\text { respetable como las relaciones entre } \\
\text { un hombre y una mujer. }\end{array}$ & 0,124 & 0,891 & 0,03 & 0,114 & 0,081 & 0,034 \\
$\begin{array}{l}\text { P604 A lo largo de la vida, una } \\
\text { persona puede variar de opción sexual }\end{array}$ & 0,076 & 0,630 & 0,105 & 0,043 & 0,081 & 0,014 \\
$\begin{array}{l}\text { y tener relaciones con hombres o con } \\
\text { mujeres. }\end{array}$ & & & & & & \\
$\begin{array}{l}\text { P605 En el fondo, la homosexualidad } \\
\text { es una enfermedad y como tal debe } \\
\text { tratarse. }\end{array}$ & 0,071 & 0,649 & 0,113 & $-0,003$ & 0,106 & 0,12 \\
& & & & & & & \\
\end{tabular}




\begin{tabular}{lcccccc}
\hline & $\mathbf{1}$ & $\mathbf{2}$ & $\mathbf{3}$ & $\mathbf{4}$ & $\mathbf{5}$ & $\mathbf{6}$ \\
\hline $\begin{array}{l}\text { P606 La relación sexual entre dos } \\
\text { hombres es una opción personal tan } \\
\text { respetable como las relaciones entre } \\
\text { un hombre y una mujer. }\end{array}$ & 0,108 & 0,900 & $-0,004$ & 0,082 & 0,074 & 0,038 \\
\hline
\end{tabular}

FACTOR 3. Sexo con o sin amor

\begin{tabular}{|c|c|c|c|c|c|c|}
\hline $\begin{array}{l}\text { P601 Para tener relaciones sexuales } \\
\text { hay que estar enamorado/a. }\end{array}$ & 0,099 & 0,177 & 0,789 & 0,116 & 0,061 & 0,086 \\
\hline $\begin{array}{l}\text { P2304 Solo puedo tener relaciones } \\
\text { sexuales con alguien si estoy } \\
\text { enamorado/a. }\end{array}$ & 0,069 & 0,121 & 0,808 & 0,177 & 0,006 & 0,074 \\
\hline
\end{tabular}

FACTOR 4. Fantasías, juguetes eróticos y porno

\begin{tabular}{|c|c|c|c|c|c|c|}
\hline $\begin{array}{l}\text { P1901 Ver espectáculos, películas, } \\
\text { leer revistas, libros de contenido } \\
\text { erótico o pornográfico. }\end{array}$ & 0,204 & 0,02 & 0,289 & 0,585 & $-0,103$ & 0,001 \\
\hline $\begin{array}{l}\text { P2805 Piensa en fantasías e imágenes } \\
\text { seductoras para aumentar su deseo y } \\
\text { excitación. }\end{array}$ & 0,077 & 0,055 & 0,099 & 0,725 & $-0,005$ & $-0,056$ \\
\hline $\begin{array}{l}\text { P2807Usa juguetes eróticos para } \\
\text { favorecer sus relaciones. }\end{array}$ & 0,092 & 0,111 & $-0,013$ & 0,626 & 0,158 & 0,026 \\
\hline
\end{tabular}

FACTOR 5. Sexualidad patriarcal/coitocentrismo

\begin{tabular}{|c|c|c|c|c|c|c|}
\hline $\begin{array}{l}\text { P2801 Toma la iniciativa en las } \\
\text { relaciones sexuales. }\end{array}$ & $-0,122$ & 0,167 & $-0,383$ & $-0,246$ & 0,404 & $-0,023$ \\
\hline $\begin{array}{l}\text { P603 Los hombres tienen más deseos } \\
\text { sexuales que las mujeres. }\end{array}$ & 0,124 & 0,057 & 0,381 & $-0,1$ & 0,427 & 0,023 \\
\hline $\begin{array}{l}\text { P2302 El sexo "de verdad" incluye la } \\
\text { penetración y el orgasmo. }\end{array}$ & 0,017 & 0,144 & 0,073 & 0,066 & 0,719 & 0,088 \\
\hline $\begin{array}{l}\text { P2802 La penetración es el objetivo } \\
\text { final de su actividad sexual. }\end{array}$ & $-0,074$ & 0,064 & $-0,056$ & 0,11 & 0,747 & 0,02 \\
\hline
\end{tabular}

FACTOR 6. Sexualidad reprimida

\begin{tabular}{lcccccc}
\hline $\begin{array}{l}\text { P2305 No me atrevo a hablar de mis } \\
\text { deseos sexuales. }\end{array}$ & 0,084 & 0,102 & 0,114 & 0,137 & 0,011 & 0,713 \\
$\begin{array}{l}\text { P2303 Mis creencias religiosas } \\
\text { influyen en la manera en que vivo mi }\end{array}$ & 0,045 & 0,335 & 0,289 & $-0,029$ & $-0,044$ & 0,416 \\
$\begin{array}{l}\text { sexualidad. } \\
\begin{array}{l}\text { P2804 Reprime e inhibe sus deseos y } \\
\text { placer sexual. }\end{array}\end{array}$ & 0,1 & $-0,005$ & $-0,038$ & $-0,143$ & 0,118 & 0,691 \\
\hline
\end{tabular}

Fuente: elaboración propia a partir de los datos del estudio n. ${ }^{\circ} 2780$ del CIS. 
Los factores se muestran en la Tabla 1 y contiene las correlaciones entre las variables originales y cada uno de los factores. Se aplicó al algoritmo de rotación varimax para facilitar la interpretación de los resultados. Las correlaciones obtenidas permiten dar contenido y capacidad interpretativa a los factores construidos (Tabla 1), asignando así una etiqueta a cada una de estas dimensiones.

Así, en la primera dimensión las variables con correlaciones más significativas son las referidas a prácticas sexuales no coitocéntricas: grado de satisfacción en su vida sexual (que le producen o le producían cuando tenía relaciones). 1) Desnudar a su pareja; 2) que su pareja le desnude, 3) acariciar a su pareja los genitales con manos, boca, lengua...; 4) que su pareja le acaricie los genitales con manos, boca, lengua...; 5) acariciar el cuerpo de su pareja con manos, boca, lengua...; 6) que su pareja le acaricie el cuerpo con manos, boca, lengua...; 7) besarse apasionadamente. Como se observa, todas estas variables abarcan una amplia gama de prácticas sexuales que tienen como común denominador el hecho de que el coito no aparece en el centro de la actividad sexual.

Con respecto a la segunda dimensión, las variables que tienen más peso en este factor son las que se refieren a actitudes hacia la diversidad en la orientación sexual: 1) la relación sexual entre dos mujeres es una opción personal tan respetable como las relaciones entre un hombre y una mujer; 2) a lo largo de la vida, una persona puede variar de opción sexual y tener relaciones con hombres o con mujeres; 3 ) en el fondo, la homosexualidad es una enfermedad y como tal debe tratarse; 4) la relación sexual entre dos hombres es una opción personal tan respetable como las relaciones entre un hombre y una mujer. Todas ellas tienen que ver con las actitudes hacia las relaciones sexuales entre personas del mismo sexo y la posibilidad de variar de orientación sexual a lo largo de la vida frente a la creencia heteronormativa en la que la heterosexualidad aparece como natural, fija y estática.

La tercera dimensión está conformada por variables referidas al sexo con o sin amor. Las variables que obtienen una carga más elevada en este factor son las siguientes: 1) para tener relaciones sexuales hay que estar enamorado/a; y 2) solo puedo tener relaciones sexuales con alguien si estoy enamorado/a. Como veíamos en la teoría, el romance heterosexual encaja dentro del modelo de amor romántico y ensalza la idea de que el sexo con amor es preferible a la versión del sexo sin amor.

En la cuarta dimensión puntúan más alto aquellas variables referidas a la frecuencia en el uso de elementos ajenos al propio cuerpo en las relaciones sexuales: 1) ver espectáculos, películas, leer revistas, libros de contenido erótico o pornográfico; 2) piensa en fantasías e imágenes seductoras para aumentar su deseo y excitación; y 3) usa juguetes eróticos para favorecer sus relaciones. En definitiva, todas aquellas variables que incor- 
poran recursos externos al propio cuerpo o al de la persona/s con la/s que se realizan las prácticas sexuales.

La quinta dimensión está formada por elementos propios de una sexualidad más masculinizada que tiene como centro el coito y que asigna al hombre la posición de sujeto activo en las relaciones sexuales: 1) el sexo "de verdad" incluye la penetración y el orgasmo; 2) la penetración es el objetivo final de su actividad sexual; 3) los hombres tienen más deseos sexuales que las mujeres; 4) toma la iniciativa en las relaciones sexuales. Se trata pues de variables que hacen referencia a una sexualidad centrada en necesidades masculinas (que tiene como centro el coito y que obvia las necesidades femeninas) y que implican la afirmación de estereotipos de género.

La última dimensión está formada por aquellas variables que se refieren a una sexualidad reprimida: 1) no me atrevo a hablar de mis deseos sexuales; 2) mis creencias religiosas influyen en la manera en que vivo mi sexualidad; 3) reprime e inhibe sus deseos y placer sexual. Aquí encontramos, como vemos, el asunto de la moral cristiana en su faceta directa (mis creencias religiosas me lo impiden) o la referencia a una sexualidad velada en la que se reprimen deseos o se ocultan los mismos.

\section{Identificación de las características demográficas que influyen en la concepción de la sexualidad}

En este apartado se trata de estudiar si existen diferencias significativas en la concepción de la sexualidad en la población española residente en función del sexo, la edad y las creencias religiosas. A partir de estas características sociodemográficas se han planteado las siguientes hipótesis a contrastar.

La primera de ellas es que, pese a que en España se han producido cambios culturales en las últimas décadas en los que a sexualidad se refiere — entre otros, la incorporación de las mujeres a diferentes aspectos de la vida-, continúan perviviendo diferencias de género que beben del profundo peso de la religión en nuestro país así como de una sociedad marcadamente patriarcal. En este sentido, y obedeciendo a esta socialización de género, las mujeres poseen una visión más normativa de la sexualidad de lo que lo hacen los hombres.

En segundo lugar, fruto de los cambios acaecidos, cabe pensar que las personas de mayor edad poseen una visión más normativa de la sexualidad de lo que lo hacen los y las más jóvenes.

Por último, atendiendo al peso de la moral judeocristiana en la concepción de la sexualidad en nuestro país, las personas católicas poseen una visión más tradicional de la sexualidad, así como las pertenecientes a otras confesiones. 
Para comprobar estas tres hipótesis se ha aplicado la técnica estadística del Análisis de Varianza (ANOVA) ${ }^{7}$, que permite contrastar la hipótesis nula de igualdad de medias en los grupos analizados. El rechazo de esta hipótesis indicará la existencia de diferencias significativas de medias en los grupos, es decir, que la variable sociodemográfica a la que pertenecen los grupos influye en la concepción de la sexualidad.

Empezando por el sexo, a la vista de los resultados, existen diferencias en cuanto a la concepción de la sexualidad entre mujeres y hombres (Tabla 2). Las mujeres obtienen puntuaciones más altas, es decir, su concepción y actitud hacia la sexualidad es más normativa en lo que respecta a las prácticas no coitocéntricas; amor romántico y las fantasías, juguetes eróticos y porno. Los hombres son más normativos en relación con la sexualidad patriarcal/coitocéntrica, así como con respecto a las actitudes hacia las orientaciones sexuales diversas (es decir, son más heteronormativos).

TABLA 2

Sexualidad ortodoxa/heterodoxa según sexo

\begin{tabular}{|c|c|c|c|c|}
\hline & \multicolumn{2}{|c|}{ Sexo } & \multirow{2}{*}{ Total } & \multirow{2}{*}{ Sig. } \\
\hline & Hombre & Mujer & & \\
\hline Prácticas no coitocéntricas & 2,165 & 2,486 & 2,313 & $0,000^{*}$ \\
\hline Actitudes hacia la orientación sexual diversa & 3,751 & 3,13 & 3,465 & $0,000^{*}$ \\
\hline Amor romántico & 4,225 & 5,476 & 4,8 & $0,000^{*}$ \\
\hline Fantasías, juguetes eróticos y porno & 7,013 & 7,428 & 7,204 & $0,000^{*}$ \\
\hline Sexualidad patriarcal/coitocetrismo & 5,507 & 4,707 & 5,139 & $0,000^{*}$ \\
\hline Sexualidad reprimida & 3,336 & 3,279 & 3,309 & $0,000^{*}$ \\
\hline
\end{tabular}

Si atendemos a la edad (Tabla 3), observamos que existen diferencias significativas entre los grupos y la concepción de la sexualidad se va haciendo más ortodoxa a medida que aumenta la edad. Esta tendencia se rompe en el caso de las actitudes hacia la orientación sexual, siendo más normativa las actitudes hacia la orientación sexual del grupo de 26 a 35 años y menos normativa la del grupo de 36 a 45 años. En el caso del uso de juguetes, también se rompe esta dinámica, siendo más normativa la concepción de los adolescentes en este aspecto. En cuanto a la sexualidad patriarcal y coitocéntrica, esta es más normativa en el grupo de 16 a 25, mientras que los dos grupos siguientes lo son un poco menos, algo que se repite en lo que se refiere a la sexualidad reprimida.

7 "El Análisis de Varianza sirve para estudiar la incidencia de una variable nominal u ordinal (categórica) con más de dos alternativas en una variable de intervalo o de razón (cuantitativa)" (Murillo y Martínez-Garrido, 2012: 53). 
TABLA 3

Sexualidad ortodoxa/heterodoxa según edad

\begin{tabular}{|c|c|c|c|c|c|c|c|c|}
\hline & \multicolumn{6}{|c|}{ Franjas de edad } & \multirow{2}{*}{ Total } & \multirow{2}{*}{ Sig. } \\
\hline & $16-25$ & $26-35$ & $36-45$ & $46-55$ & $56-65$ & 65 o más & & \\
\hline $\begin{array}{l}\text { Prácticas no } \\
\text { coitocéntricas }\end{array}$ & 2,203 & 2,025 & 2,223 & 2,442 & 2,808 & 3,039 & 2,313 & $0,000 *$ \\
\hline $\begin{array}{l}\text { Actitudes hacia la } \\
\text { orientación sexual diversa }\end{array}$ & 3,116 & 3,275 & 3,197 & 3,569 & 3,985 & 5,086 & 3,465 & $0,000^{*}$ \\
\hline Amor romántico & 4,617 & 4,683 & 4,803 & 4,901 & 5,102 & 5,063 & 4,800 & $0,000 *$ \\
\hline $\begin{array}{l}\text { Fantasías, juguetes } \\
\text { eróticos y porno }\end{array}$ & 7,178 & 7,027 & 7,084 & 7,293 & 7,541 & 7,695 & 7,204 & $0,000^{*}$ \\
\hline $\begin{array}{l}\text { Sexualidad patriarcal/ } \\
\text { coitocetrismo }\end{array}$ & 5,152 & 5,017 & 5,051 & 5,174 & 5,417 & 5,406 & 5,139 & $0,000 *$ \\
\hline Sexualidad reprimida & 3,246 & 3,216 & 3,216 & 3,320 & 3,480 & 3,902 & 3,309 & $0,000^{*}$ \\
\hline
\end{tabular}

Según la creencia religiosa declarada por la persona entrevistada, la tendencia es que las personas creyentes poseen una concepción más normativa de la sexualidad que las que no lo son. Las y los católicos son más normativos que los creyentes de otras religiones en cuanto a las prácticas no coitocéntricas y al empleo de juguetes. Sin embar-

Tabla 4

Sexualidad ortodoxa/heterodoxa según creencias religiosas

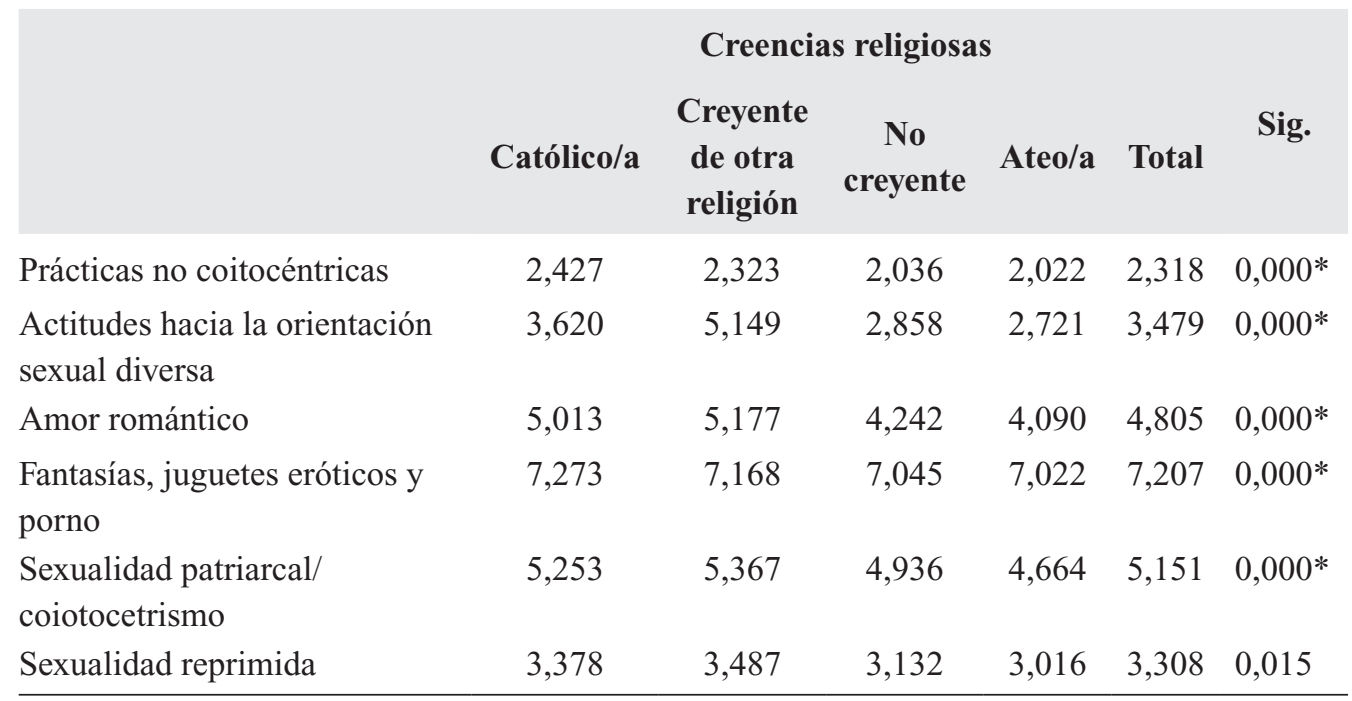


go, los creyentes de otra religión muestran una diferencia más acentuada con las personas católicas en lo que se refiere a las actitudes hacia la orientación sexual. En este sentido, sería interesante ver qué confesiones predominan en la población residente española y si esta muestra permite hacer inferencias sobre las razones de estas diferencias. Se repite esto último aunque con diferencias menos acentuadas en lo que respecta a sexualidad patriarcal, sexualidad reprimida y amor romántico.

\section{CONCLUSIONES}

En cuanto a los resultados obtenidos, en esta primera aproximación podemos extraer la conclusión de que sí existen diferencias en mujeres y hombres, en personas de diferentes edades y con diversas creencias religiosas en lo que respecta a las concepciones sobre la sexualidad.

Las mujeres en su conjunto poseen un concepto de la sexualidad más normativo salvo en aspectos como la "orientación sexual diversa" y "la sexualidad patriarcal/coitocéntrica", en la que son los hombres quienes manejan concepciones más tradicionales. Como apuntaban nuestras hipótesis, a pesar de los profundos cambios acaecidos en las últimas décadas en nuestro país, lo cierto es que la socialización de género se enmarca dentro de una sociedad patriarcal que sigue profundizando en las diferencias de género basadas en presuntas bases biológicas. Estos guiones culturales de género acaban, a la luz de los resultados, por poseer un gran peso en los comportamientos y las actitudes sexuales de las españolas en este intercambio entre guiones culturales/intrapsíquicos/ interpersonales.

Las mujeres, en esta primera aproximación, presentan una actitud más tolerante en cuanto a las actitudes hacia la orientación diversa. Ayuso y García (2014) apuntaban al amplio consenso sobre la tolerancia en aumento de los últimos años sobre las relaciones entre personas del mismo sexo. No obstante, pese a esta tendencia, lo cierto es que existen diferencias por género en este sentido. Dentro del esquema de la heterosexualidad obligatoria (y de sus guiones), la masculinidad hegemónica presentaría rasgos menos flexibles con respecto a la tolerancia hacia la diversidad en la orientación sexual. Por otro lado, las mujeres otorgan un peso más reducido al coito y a su vez desarrollan una actitud más pasiva en el terreno sexual. Resultado que, en conjunto, corrobora la representación de un guion sexual más activo en el caso de los hombres y, en contraposición, un rol femenino más pasivo (Frith y Kitzinger, 2001; Alksnis, 1996; Macionis y Plummer, 2012: 352-353).

Con respecto a la edad, el patrón dibujado es que a menor edad, una concepción más ortodoxa y tradicional de la sexualidad. La "transición sexual” (Ayuso y García, 2014) 
es más palpable en el aspecto de la edad y camina en sintonía con estudios previos (Ayuso y García, 2014; INJUVE, 2011) que apuntaban a una diferencia generacional y a una tendencia hacia la aceptación de una mayor plasticidad de las identidades sexuales y de un mayor reconocimiento de la existencia de la diversidad afectivo-sexual.

En cuanto a la religión, las personas católicas o creyentes de otra religión son más normativas que las no creyentes o ateas. Cabe mencionar que la diferencia más profunda entre católicos/as y creyentes de otra religión se da en lo que respecta a las "actitudes hacia la orientación sexual diversa", siendo más ortodoxa la concepción de los creyentes de otra religión. En futuros análisis que ahonden en este aspecto convendría cuantificar qué otras confesiones están incluidas en las entrevistas y otorgar una explicación fundamentada a este respecto.

Visto lo anterior, podemos afirmar que en la actualidad en nuestro país conviven concepciones más tradicionales de la sexualidad frente a otras más heterodoxas en función de diferentes rasgos sociodemográficos. No obstante, se trata de una primera aproximación y cabría realizar en un futuro análisis más exhaustivos con el objetivo de comprobar las hipótesis propuestas y aportar mayor robustez metodológica. Por tanto, lo valioso del análisis realizado en el presente trabajo es que el modelo factorial esbozado otorga una herramienta para medir cómo es la sexualidad. Una vez hemos diseñado este modelo factorial de la sexualidad, podemos realizar con el mismo todos aquellos análisis que estimemos oportunos. Así pues, de lo que no cabe duda es de que este trabajo representa un primer análisis de otros ulteriores con el objetivo de sacar rendimiento y aprovechar el modelo factorial propuesto, algo inexistente hasta el momento.

Por tanto, la relevancia de este trabajo radica en hacer operativo el concepto de sexualidad y dotar de un instrumento que nos permita hacer medible algo que hasta ahora no se había medido, los modelos de sexualidad normativa/no-normativa en España. Por ello, el presente trabajo representa una importante contribución al estudio de la sexualidad, que puede servir a futuras investigaciones que quieran transitar esta senda y profundizar en este campo, ampliando así el conocimiento en el área de la sociología de la sexualidad.

\section{BIBLIOGRAFÍA}

Alksnis, C., Desmarais, S. y Wood, E. (1996). "Gender differences in scripts for different types of dates". Sex Roles, 34 (5-6): 321-336. https://doi.org/10.1007/ bf01547805

Ayuso Sánchez, L. (2015). "La sexualidad en las relaciones íntimas”, en Torres Albero, Cristóbal, España 2015, situación social, Madrid: Centro de Investigaciones Sociológicas. 
Ayuso Sánchez, L. y García Faroldi, L. (2014). Los españoles y la sexualidad en el siglo XXI (No.281). Madrid: Centro de Investigaciones Sociológicas.

Bersntein, M. (2013). "The sociology of Sexualities: Taking Stock of the Field". Contemporary Sociology, 42, 1 (January 2013): 22-31.

CIS, el Observatorio de Salud de la Mujer, Encuesta Nacional de Salud Sexual. Estudio n. ${ }^{\circ}$ 2780. Madrid, 2009, disponible en http://www.cis.es/cis/opencm/ES/1_encuestas/estudios/ver.jsp?\&estudio $=9702$

CIS (2009). Informe metodológico. Encuesta Nacional de Salud Sexual. Estudio n. ${ }^{\circ}$ 2780. Madrid: Centro de Investigaciones Sociológicas.

De Ussel, J.I. (1983). “La sociología de la sexualidad en España: Notas introductorias”. Reis, 21(21): 103-133. https://doi.org/10.2307/40183001

Frith, H. Y Kitzinger, C. (2001). "Reformulating sexual script theory: Developing a discursive psychology of sexual negotiation". Theory and psychology, 11(2): 209232. https://doi.org/10.1177/0959354301112004

Gagnon, J. H. y Simon, W. (1973). Sexual conduct: The social sources of human sexuality. Chicago: Aldine.

Guasch, O. (1993). "Para una sociología de la sexualidad". Reis, 64(64): 105-121. https://doi.org/10.2307/40183700

Giddens, A. (1995). La transformación de la intimidad: Sexualidad, amor y erotismo en las sociedades modernas. Madrid: Cátedra.

INJUVE (2011). Sondeo de opinión "Jóvenes y diversidad sexual”. Conclusiones. Ministerio de Sanidad, Política Social e Igualdad.

Laumann, E.O., Gagnon, J.H., Michael, R.T., y Michaels, S. (2000). The social organization of sexuality: Sexual practices in the United States. Chicago: University of Chicago Press.

Macionis, J. J., y Plummer, K. (2012). Sociología (4. ${ }^{\text {a }}$ ed.). Madrid [etc]: Prentice-Hall. MacKinnon, C. A. (1979) Sexual harassment of working women: A case of sex discrimination. New Haven: Yale University Press.

Masters, W.H., Johnson, V.E., y Kolodny, R.C. (1995). La sexualidad humana. 3. Perspectivas clínicas y sociales. Barcelons: Grijalbo.

Miguel, A. de (1975). Sexo, mujer y natalidad en España. Madrid: Cuadernos para el Diálogo.

Millett, K., y Moreno, A. 1. (1995). Política sexual. Madrid. Valencia: Cátedra.

Moreno, B., y Magdaleno Dans, F. (1990). La sexualidad humana: Estudio y perspectiva histórica. Madrid: Fundación Universidad-Empresa. 
Murillo Torrecilla, F. J., y Martínez-Garrido, C. (2012). Análisis de datos cuantitativos con SPSS en investigación socioeducativa. Madrid: Ediciones de la Universidad Autónoma de Madrid.

Osborne, R., y Guasch, O. (2003). Sociología de la sexualidad. Madrid: Centro de Investigación Sociológicas.

Rubin, Gayle (1989): "Reflexionando sobre el sexo. Notas para una teoría radical de la sexualidad", en Carole S. Vance (comp.), Placer y peligro. Explorando la sexualidad femenina. Madrid: Talasa.

Simon, W., y Gagnon, J.H. (2003). "Sexual scripts: Origins, influences and changes". Qualitative Sociology, 26(4): 491-497. https://doi.org/10.1023/b:quas. 0000005053.99846.e5

- (2011) Sexual Conduct: The Social Sources of Human Sexuality. Transaction Publishers.

Stein, A. y Plummer, K. (1994). «"I Can’t Even Think Straight”: "Queer” Theory and the Missing Sexual Revolution in Sociology». Sociological Theory, 12, 2 (July 1994): 178-187. https://doi.org/10.2307/201863

Wiederman, M.W. (2015). "Sexual Script Theory: Past, Present, and Future”, en DeLamater, J., Plante, R.F., \& SpringerLink (Online service). Handbook of the sociology of sexualities (2015th ed.). Cham: Springer International Publishin. https://doi. org/10.1007/978-3-319-17341-2_2 